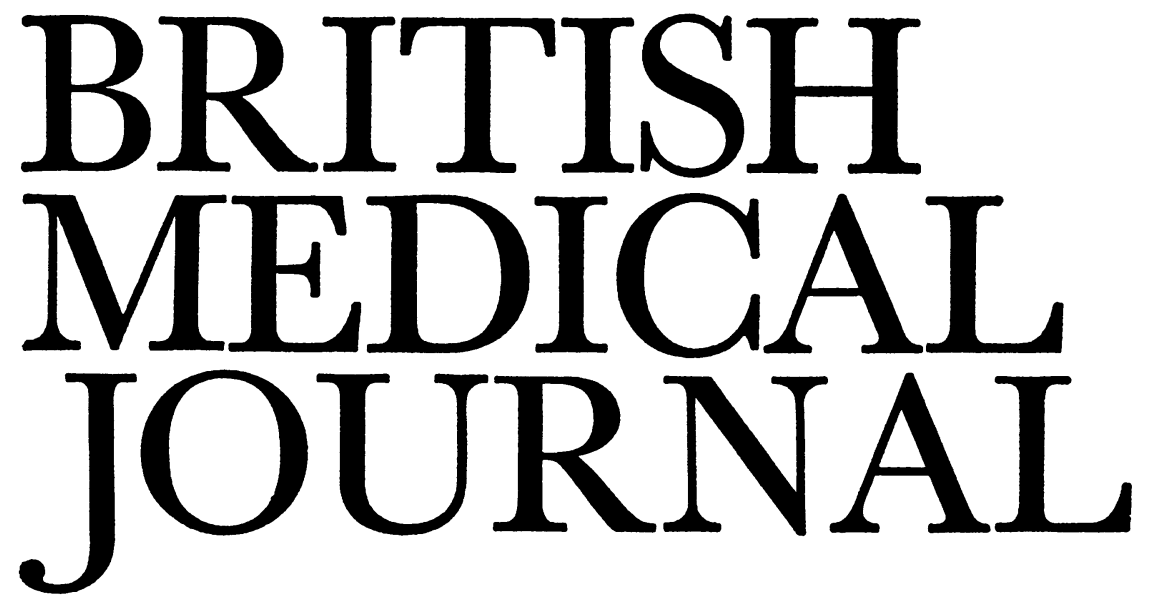

\title{
Meningococcal Infections
}

Meningococcal meningitis seems to be on the increase in Britain. The rate of notifications recorded by the Registrar General $^{1}$ has been rising since about 1967 and the number of infections reported each year to the Public Health Laboratory Service has also risen ${ }^{2}$-from 358 in 1967 to 843 in 1973 (see p. 355). Meningitis is not usually thought of nowadays as an epidemic disease, but outbreaks sometimes occur in families and in schools or military recruit camps. Most notifications in the United Kingdom represent single, sporadic cases, but whenever meningococcal infection is diagnosed the doctor has to consider the possibility of infection among contacts of his patient and the need for measures to prevent further cases.

Meningococci usually exist harmlessly in the nasopharynx. A recent study from East $\mathrm{Anglia}^{3}$ reported that the carrier rate in persons of all ages was $21 \%$ and among 15 to 16-year-old naval recruits, swabbed on first joining a training establishment, the rate was $30 \%$. After nine months in the camp $71 \%$ were carriers, but there was only one clinical case of infection, which occurred during a leave period. Meningococci are thus common in the normal nasopharynx and readily spread under conditions of close contact, but illness is rarely caused. Part of the explanation is naturally acquired immunity ${ }^{4-6}$ resulting from the carriage of strains of meningococci of low virulence. In groups of persons exposed to virulent meningococci many will be immune, particularly in the older age groups, and cases are likely to arise only in those who have no previously acquired natural immunity.

The possibility of vaccinating against infection is under study in the U.S.A. Meningococci fall into a number of serological groups, of which $\mathrm{A}, \mathrm{B}$, and $\mathrm{C}$ are the most prevalent in clinical cases. Group B is nowadays the most common in Britain, with group $\mathrm{C}$ strains next in importance and group A relatively uncommon. Purified carbohydrate vaccine prepared from group $\mathrm{C}$ meningococci has been shown to protect against group $C$ infection in field trials, ${ }^{7}$ but as yet no satisfactory group B vaccine has been prepared. However, meningococcal infection is still a rare disease in Britain, and the case for using a group $C$ vaccine (or even a $B$ vaccine if it were available) is not strong. Moreover, prophylaxis is possible with antimicrobial agents.

The aim of chemoprophylaxis is to remove the meningococci from the nasopharynx of everyone in a population at risk, thereby protecting individuals lacking natural immunity. For many years sulphonamides have been effective in prophylaxis, ${ }^{8}$ but resistant strains were reported ${ }^{9}$ in 1963 in the U.S.A. and nowadays most group $\mathrm{C}$ isolates in that country are resistant. ${ }^{10}$
In Britain, too, resistance has appeared, and in the past two to three years about $10-15 \%$ of strains have been found $\mathbf{3}^{\mathbf{1} 11^{-13}}$ to be fully resistant, but in Scotland in the first quarter of 1974 it was found that an additional $52 \%$ of strains were partially resistant. ${ }^{14}$ Sulphonamides alone should therefore no longer be used to start treatment in clinical cases, nor can they be relied on for prophylaxis unless there is good reason to believe that the infecting strains are sensitive. Penicillins-and a variety of other antimicrobials to which meningococci are sensitive-are inefficient in eliminating the organisms from the nasopharynx. ${ }^{15}$ Rifampicin is effective, but resistant meningococci may appear rapidly in the population in which it is used. ${ }^{1617}$ Minocycline, an antibiotic related to tetracycline, has also been found to be reasonably effective ${ }^{1518}$ in clearing carriers in a military station, but experience with it is limited. However, the wide use of an antimicrobial among contacts of a case of meningitis should not be advocated lightly. Not only may resistant strains be encountered, they may be encouraged; while the security given by prophylaxis may be short-lived because meningococci are apt to be reintroduced into a family or group when the treatment is finished.

What measures, then, can be recommended to the doctor confronted with meningococcal meningitis or bacteraemia in a family or school ? A guide with which all experts would agree probably cannot be prepared at the present time, but a reasonable approach can be suggested. The patient requires a lumbar puncture and also blood culture for firm diagnosis and to identify the infecting organism and its sensitivity to antimicrobial drugs. Most patients should, therefore, be admitted to hospital, which has the added effect of removing a source of virulent infection from the family. The infection should be notified-an important step as it allows the medical officer of environmental health to spot quickly the occurrence of more than one case in a locality; he is then able to take further action, such as warning other family doctors or, perhaps, dealing with a school outbreak. The family and other close contacts known to the doctor should be kept under close surveillance, so that any further cases will be diagnosed and treated early. The family should be asked to see that all illnesses, including mild indisposition, are reported immediately, and a daily visit from a health visitor would be a valuable help in this. Exclusion of family contacts from school may also be considered.

The occurrence of more than one case in a family or group of families in close contact or in an institution should be regarded more seriously, as should a single case in a family 
with a number of children living in overcrowded conditions. ${ }^{20} 21$ Efforts should be made to diminish close contact within the group concerned, and also with other families or schools, and closure of an affected institution may sometimes be necessary. It is mainly in these more serious situations that chemoprophylaxis should be considered. If the meningococcus isolated from the original case is sensitive to sulphonamides, or believed likely to be so, prophylaxis may be practised by simultaneously treating the whole group with a two-day course of sulphadiazine. ${ }^{320}$ If the meningococcus is sulphonamide-resistant the use of an alternative drug such as minocycline may be justified, preferably under close bacteriological and clinical supervision so that the effects may be documented and reported for the guidance of others. Chemoprophylaxis need not be dependent on culture of nasopharyngeal swabs from contacts, but if facilities are available control of the outbreak may be helped by identifying carriers and the antimicrobial sensitivity of the meningococci isolated.

1 Lambert, P. M., Community Medicine, 1973, 129, 279.

2 Public Health Laboratory Service, British Medical fournal, 1974, 2, 453.

3 Fraser, P. K., et al., Lanc.t, 1973, 1, 1235.

Goldschneider, I., Gotschlich, E. C., and Artenstein, M. S., Fournal of Experimental Medicine, 1969, 129, 1307 5 Goldschneider, I., Gotschlich, E. C., and Artenstein, M. S., Fournal of

${ }^{6}$ Reller, L. B., MacGregor, R. R., and Beaty, H. N., Fournal of Infectious Diseases, 1973, 127, 56 .

Artenstein, M. S., et al., New England Fournal of Medicine, 1970, 282, 417.

8 Kuhns, D. M., et al., Fournal of the American Medical Association, 1943, $123,335$.

${ }^{9}$ Millar, J. W., et al., Fournal of the American Medical Association, 1963, 186, 139.

${ }^{10}$ Fraser, D. W., Thornsberry, C., and Feldman, R. A., fournal of Infectious Diseases, 1972, 125, 443.

11 Abbott, J. D., and Graves, J. F. R., Fournal of Clinical Pathology, 1972, 25, 528.

12 Easton, D. M., et al., British Medical fournal, 1974, 1, 507.

13 Fallon, R. J., Communicable Diseases Scotland, 23 February 1974.

14 Fallon, R. J., Communicable Diseases Scotland, 11 May 1974.

15 Fallon, R. J., Communicable Diseases Scotland, 11 May 1974.

16 O'Beirne, A., and Robinson, J. A., American Fournal of Medical Science, $1971,262,33$.

17 Beam, W. E. Jr., et al., Fournal of Infectious Diseases, 1971, 124, 39.

18 Devine, L. F., et al., American fournal of Epidemiology, 1971, 93, 337.

10 Guttler, R. B., and Beaty, H. N., Antimicrobial Agents and Chemotherapy, $1972,1,397$.

20 Sharpe, W., et al., Community Medicine, 1972, 129, 211.

21 Sanders, E., and Deal, W. B., Fournal of Infectious Diseases, 1970, 121, 449.

\section{Renal Vascular Damage after Birth}

In the neonatal period the kidney seems unusually prone to vascular damage from thrombosis, embolism, or ischaemic necrosis. The factors responsible for this susceptibility include the small calibre of the renal vessels, the low renal blood flow, the rapid rate of fluid turnover, and the relative polycythaemia of the newborn period. In addition there are specific causes including perinatal asphyxia, birth trauma, blood loss, saline depletion or hyperosmolar dehydration following diarrhoea, septicaemia with disseminated intravascular coagulation, cyanotic heart disease, radiological studies utilizing contrast media, ${ }^{12}$ and maternal diabetes.

The most common form of vascular abnormality is renal venous thrombosis ${ }^{3}$ (the preferred terminology for the usual site of the thrombosis) in the arcuate or interlobular veins with extension to the cortex or to the intralobular and main renal veins. ${ }^{4}$ Unilateral and bilateral cases occur with equal frequency, ${ }^{5}$ usually in the first week of life, but a recent European survey showed that nearly half the cases were discovered only at necropsy, ${ }^{4}$ suggesting the need for greater awareness of conditions predisposing to the condition and of its clinical features. Haematuria and enlarged palpable kidneys are found in more than half the cases, but absence of these signs does not preclude the diagnosis: oliguria, uraemia, metabolic acidosis, proteinuria, anaemia, and thrombocytopenia may be present. The last two findings suggest the possibility of a consumptive coagulopathy and microangiopathic haemolytic anaemia as a secondary or even predisposing phenomenon to the venous thrombosis. ${ }^{6}$

Oedema and cyanosis of the legs may be found if there is thrombosis of the inferior vena cava. Intravenous urography is the most useful investigation, but it should be postponed until the clinical condition has improved after appropriate resuscitation. It is better undertaken by units with special experience of renal radiography in this age group, for it is not without risk, ${ }^{2}$ and the interpretation may be difficult. The finding of an enlarged, poorly functioning kidney with no pelvicaliceal dilatation sustains the clinical diagnosis, but if a nonfunctioning kidney is present then further investigations such as nephrosonography, ${ }^{7}$ antegrade pyelography, ${ }^{8}$ or retrograde pyelography may be required to exclude renal agenesis, cystic dysplasia, or hydronephrosis. Final confirmation, especially in bilateral cases, requires inferior vena caval and selective renal vein venography, ${ }^{9}$ but the risks deter most paediatricians unless thrombectomy is contemplated.

Reports that the condition is invariably fatal must now be revised: over one third of the children reported in the European survey survived in good health. Conservative management includes peritoneal dialysis where necessary. Heparin anticoagulation is commonly used, ${ }^{4}$ especially where consumptive coagulopathy coexists, though no controlled trial has been reported to justify it and recovery is possible without heparin, even in bilateral cases. ${ }^{10}$ Thrombectomy for bilateral cases with vena caval thrombosis has been successful, ${ }^{11} 12$ but again the treatment is controversial. ${ }^{913}$ For the present it seems reasonable not to commend thrombectomy until an adequate trial has been concluded, though such a study would require co-operation between a number of centres.

The recognition of survivors has led to reports of late sequelae; hypertension may appear some months later, and possibly non-functioning kidneys should be removed, after the acute stage but before hypertension develops. ${ }^{4}$ Other sequelae include the nephrotic syndrome, ${ }^{14}$ while recently multiple renal tubular abnormalities were found in three children who had evidence of neonatal renal venous thrombosis. ${ }^{15}$ One of the children had a classical Fanconi syndrome; it seems that renal vascular accidents must now be added to the list of acquired and congenital causes of this syndrome.

Adrenal haemorrhage ${ }^{16}$ is uncommon, but when it occurs it may be confused with renal venous thrombosis. The onset is sudden with pallor, lethargy, and shock; a large mass is often palpable in the loin and there may be blood-stained ascites. Oliguria, proteinuria, and microscopic haematuria occur, and intravenous urography shows the kidney displaced downwards with compression of upper pole calices. The haemorrhage rapidly calcifies, and this can be noted on plain radiographs 2-4 weeks later. Temporary adrenal insufficiency may be found in bilateral cases. Renal arterial thrombosis ${ }^{5}$ is rare and usually secondary to embolism from bacterial endocarditis; it may be an incidental complication of congenital heart disease, umbilical arterial catheterization, ${ }^{17}$ or renal venous thrombosis. The diagnosis is difficult, but evidence of arterial thrombosis remote from the kidneys should be sought.

Severe cases of acute tubular necrosis may be accompanied by papillary necrosis ${ }^{\mathbf{1 8}}$ and again the precipitating factors are similar to those in renal venous thrombosis. Late effects 